\title{
The role of salvage surgery in oral squamous cell carcinoma
}

\section{Raúl González-García}

Department of Oral and Maxillofacial-Head and Neck Surgery, University Hospital Infanta Cristina, 06080 Badajoz, Spain. Correspondence Author: Prof. Raúl González-García, Department of Oral and Maxillofacial-Head and Neck Surgery, University Hospital Infanta Cristina, 06080 Badajoz, Spain. E-mail: raulmaxilo@gmail.com

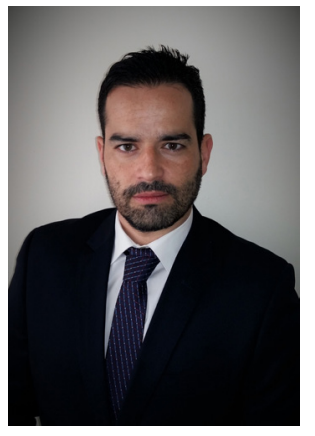

Prof. Raúl González-García, M.D., Ph.D, FEBOMFS, was graduated in Medicine (Medical Degree, M.D.) by the Universidad Autónoma de Madrid School of Medicine, Madrid, Spain, in 2001, and obtained his trainee in Oral and Maxillofacial Surgery in the University Hospital La Princesa, Madrid, Spain, in 2007. He obtained his Ph.D. Degree in Medicine and Surgery at the Universidad Autónoma de Madrid School of Medicine, in 2011. Since 2007 he is Consultant Surgeon at the Department of Oral and Maxillofacial Surgery, University Hospital Infanta Cristina, Badajoz, Spain, and he is also a University Honorary Collaborator at the Universidad de Extremadura School of Medicine. He is actually Editor-in-Chief of Plastic and Aesthetic Research, Assistant Director of Revista Española de Cirugía Oral y Maxilofacial, Permanent Member of the Reviewer Commitee of International Journal of Oral and Maxillofacial Surgery, Contributing Editor of Current Research in Dentistry, and Member of the Editorial Board/reviewer of more than 35 international journals of the speciality. He is author of more than 130 publications, 96 of them in international Medline/PubMedindexed journals, 15 chapters in books of the speciality, and he is author of the text book "Reconstruccion Maxilomandibular Compleja: Microcirugía, Distracción Ósea e Implantes dentales".

\section{ABSTRACT}

Aim: To select and analyze the most representative papers published in the literature concerning oral squamous cell carcinoma (OSCC), specifically dealing with salvage surgery following primary treatment by surgery with or without by postoperative radiotherapy, specifically focusing in the oral cavity and oropharynx locations. Methods: A bibliography search on MEDLINE and EMBASE databases for studies published from March 2000 to March 2016 was conducted. The authors only included studies published in the English language and those dealing with "squamous cell carcinoma of the oral cavity and/or oropharynx". The following technical bibliographic exclusion criteria were applied: (1) case reports; (2) technical report; (3) animal or in vitro studies; (4) review articles; (5) uncontrolled clinical studies; and (6) publications in which the same data were published by the same group of researchers. The abstracts of yielded results were reviewed and the full text of those with apparent relevance was obtained. Results: A total amount of 188 studies were found using the above reported searching parameters. Thirteen original papers were finally selected according to the inclusion and exclusion criteria. From 1,692 analyzed patients, overall recurrence rate was $26 \%$ (range: $15-41.7 \%$ ), with a mean $47.3 \%, 35.1 \%$ and $10.9 \%$ local, regional and loco-regional recurrence, respectively. Mean 5-year overall survival rate was 40.2\% (range: $37.5-42.9 \%$ ). Conclusion: Salvage surgery is the best option for the treatment of recurrent OSCC, either local, regional or loco-regional, with the highest rates in terms of survival and with an acceptable morbidity.

\section{Key words:}

Salvage surgery; oral squamous cell carcinoma; recurrent oral cancer

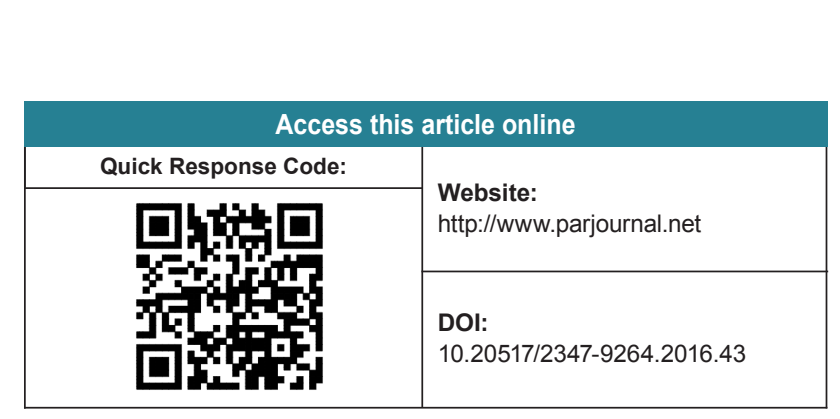

This is an open access article distributed under the terms of the Creative Commons Attribution-NonCommercial-ShareAlike 3.0 License, which allows others to remix, tweak and build upon the work non-commercially, as long as the author is credited and the new creations are licensed under the identical terms.

For reprints contact: service@oaepublish.com

How to cite this article: González-García R. The role of salvage surgery in oral squamous cell carcinoma. Plast Aesthet Res 2016;3:189-96.

Received: 07-06-2016; Accepted: 17-06-2016 


\section{INTRODUCTION}

Nowadays, recurrence in oral squamous cell carcinoma (OSCC) remains the main cause for failure in oral cancer patients, despite advances in surgical techniques and chemo-radiotherapy (CRT) protocols. In fact, treatment of OSCC with radical surgery followed by radiotherapy (RT) has been reported to be unsuccessful in $25 \%$ to $48 \%$ of the cases. ${ }^{[1]}$ This recurrence can be local-oral cavity or oropharynx-, regional- in the neck, ipsilateral, contralateral or both-, or loco-regional. Today, salvage surgery is generally considered to be the best choice for treating recurrent OSCC patients that have been previously irradiated, although cure rates are still poor. Also, when dealing with regional recurrences, primary treatment seems to determine overall outcome following salvage surgery, with poorer rates in previously irradiated and surgically treated necks. ${ }^{[2]}$

Otherwise two conditions are mandatory for this subset of patients to undergo salvage surgery: (1) the recurrence has still to be resectable in terms of resectability criteria within the head and neck region; and (2) the patient has to be operable in terms of an aesthesiologic critera. Other eligible options are generally behind in terms of curative intention such as re-irradiation or CRT, while palliative chemotherapy and supportive care are reserved for noncurative patients.

In considering the treatment to be performed, especially for those patients with advanced recurrences, there is a trend to outweigh the importance of quality of life for the patient, even more than in the first approach to the disease, in which survival is far from any other consideration, such as function and aesthetic impairment. Moreover if one takes into consideration that many of these advanced recurrent patients may undergo a permanent tracheostomy and/or a gastrostomy, a realistic evaluation of the probability of cure versus the generated morbidity has to be evaluated and sincerely approached with the patient and his/her family before salvage surgery is to be offered for the recurrent patient.

The purpose of the present study was to select and analyze the most representative papers published in the literature concerning OSCC, specifically dealing with salvage surgery following primary treatment by surgery with or without by postoperative radiotherapy, specifically focusing in the oral cavity and oropharynx locations.

\section{METHODS}

A bibliography search on MEDLINE and EMBASE databases for studies published from March 2000 to March 2016 was conducted, with the searching terms: (("salvage therapy" [MeSH Terms] OR ("salvage" [All Fields] AND "therapy" [All Fields]) OR "salvage therapy" [All Fields] OR "salvage" [All Fields]) AND ("surgery, oral" [MeSH Terms]
OR ("surgery" [All Fields] AND "oral" [All Fields]) OR "oral surgery" [All Fields] OR ("surgery" [All Fields] AND "oral" [All Fields]) OR "surgery, oral" [All Fields]) AND ("carcinoma, squamous cell" [MeSH Terms] OR ("carcinoma" [All Fields] AND "squamous" [All Fields] AND "cell" [All Fields]) OR "squamous cell carcinoma" [All Fields] OR ("squamous" [All Fields] AND "cell" [All Fields] AND "carcinoma" [All Fields])). References were explored to identify other articles.

A total amount of 188 studies were found using the above reported searching parameters. We only included studies published in the English language and those dealing with "squamous cell carcinoma of the oral cavity and/or oropharynx", excluding those exclusively referring to the larynx, hypopharynx or other sites of the head and neck, such as paranasal sinuses or salivary gland neoplasms. If an overall approach to multiple locations of the upper aero-digestive tract was performed in a particular study, only those locations referred to the oral cavity and/or oropharynx were taken into consideration regarding data output, if available. However, if no specific data was offered for "oral cavity" and "oropharynx" and only general data was offered, then the series was rejected for inclusion in the meta-analysis. Articles dealing with CRT as the salvage treatment of choice for recurrent tumors were also excluded from this review. Besides, articles specifically focusing on the reliability of the used reconstructive method or surgical technique were not considered if data referring to prognosis and/or survival analysis was not performed.

Then, a manual screening of articles' abstracts was performed in order to explore the role of salvage surgery in squamous cell carcinoma of the oral cavity and oropharynx from the ultimate complete 16 years. The following technical bibliographic exclusion criteria were applied: (1) case reports; (2) technical reports; (3) animal or in vitro studies; (4) review articles; (5) uncontrolled clinical studies; and (6) publications in which the same data were published by the same group of researchers. The abstracts of yielded results were reviewed and the full text of those with apparent relevance was obtained. The references of identified articles were crosschecked for unidentified articles. The author carefully assessed the eligibility of all studies retrieved from the databases. A total amount of 13 original papers were finally selected according to the provided inclusion and exclusion criteria.

\section{RESULTS}

\section{Summary of results from the selected studies}

From the review of the selected papers, several common weakness among them have to be highlighted, such as the difficulty in establishing prospective series of patients submitted for different treatment modalities, the variation in the series' size, and the lack of homogeneity 
Table 1: Selected studies from systematic review and meta-analysis

\begin{tabular}{|c|c|c|c|c|c|c|c|}
\hline & $\begin{array}{l}\text { Recurrent } \\
\text { patients'series } \\
\text { size }(n)\end{array}$ & $\begin{array}{l}\text { Overall } \\
\text { series } \\
\text { size }(n)\end{array}$ & $\begin{array}{l}\text { Overall } \\
\text { recurrence } \\
\text { rate }(\%)\end{array}$ & $\begin{array}{l}\text { Local } \\
\text { recurrence } \\
n(\%)\end{array}$ & $\begin{array}{l}\text { Regional } \\
\text { recurrence } \\
n(\%)\end{array}$ & $\begin{array}{l}\text { Loco-regional } \\
\text { recurrence } \\
n(\%)\end{array}$ & $\begin{array}{c}\text { 5-year overall survival } \\
\text { after salvage surgery } \\
(\%)\end{array}$ \\
\hline Schwartz et al. ${ }^{[3]} 2000$ & 38 & $135^{\star}$ & $28 \%$ & - & - & - & $21 \%$ \\
\hline Kowalski $^{[4]} 2002$ & 214 & 513 & $41.7 \%$ * & - & $82(38.3 \%)$ & - & $31-36 \%$ \\
\hline Lin et al. ${ }^{[1]} 2004$ & 56 & 191 & $29.3 \%$ * & - & - & - & $38-60 \%$ \\
\hline Agra et al. ${ }^{[5]} 2006$ & 246 & - & - & $154(62.6 \%)$ & $59(24 \%)$ & $33(13.4 \%)$ & $32 \%$ \\
\hline Koo et al. ${ }^{[6]} 2006$ & 36 & 127 & $28 \%$ & $15(41.7 \%)$ & $13(36.1 \%)$ & $3(8.3 \%)$ & $38 \%$ \\
\hline Brown et al..$^{[7]} 2007$ & 98 & 462 & $21 \%$ & $48(59.2 \%)$ & $34(34.7 \%)$ & $16(16.3 \%)$ & - \\
\hline Liao et al. ${ }^{[8]} 2008$ & 272 & 953 & $28.5 \%$ & $133(48.9 \%)$ & $139(51.1 \%)$ & - & $36 \%$ \\
\hline Lim and Choi ${ }^{[9]} 2008$ & 16 & 76 & $21 \%$ & $5(31.2 \%)$ & $8(50 \%)$ & $2(12.5 \%)$ & $36 \%$ \\
\hline Zafereo et al. ${ }^{[17]} 2009$ & 434 & 1681 & $26 \%$ & $199(45.8 \%)$ & $53(12.2 \%)$ & - & $28 \%$ \\
\hline Kernohan et al. ${ }^{[10]} 2010$ & 117 & 533 & $22 \% *$ & $39(33.3 \%)$ & $38(32.5 \%)$ & - & $50 \%$ \\
\hline Sklenicka et al. ${ }^{[11]} 2010$ & 24 & 157 & $15 \%$ & $11(45.8 \%)$ & $9(37.5 \%)$ & $1(4.1 \%)$ & $48 \%$ \\
\hline Kostrzewa et al. ${ }^{[12]} 2010$ & 72 & - & - & - & - & - & $44 \%$ \\
\hline Goto et al. ${ }^{[13]} 2016$ & 69 & - & - & - & - & - & $48-86 \%$ \\
\hline $\begin{array}{l}\text { Overall results }^{a} \\
\text { (González-García, } \\
\text { 2016) }\end{array}$ & 1,692 & - & $26 \%$ & $47.3 \%$ & $35.1 \%$ & $10.9 \%$ & $\begin{array}{c}40.2 \% \\
(37.5-42.9 \%)\end{array}$ \\
\hline
\end{tabular}

*Indirectly calculated by the author from data provided in respective publications; ${ }^{\text {a }}$ Overall results from recalculation of variables in previous analyzed studies, considering only articles with available data in relation to each particular variable

for inclusion/exclusion criteria and treatment protocols among institutions. All these features make quantitative analysis of results difficult if bias wants to be dismissed. The following paragraphs in the results section will deal with the description of the main results provided by the authors of the 13 selected papers in a chronologic manner [Table 1]. Further qualitative analysis of these results will be individually approached in the discussion section.

Up in the beginning of the 21st century, Schwartz et al.$^{[3]}$ in a retrospective study about 38 patients that had developed recurrence of oral cavity SCC, reported an overall recurrence rate of $28 \%$, with a local recurrence of $58 \%$, a loco-regional recurrence of $27 \%$ and an isolated regional recurrence of $16 \%$. With an overall salvage cure rate of $21 \%$, they found that those patients receiving surgery as salvage treatment modality significantly improved in terms of survival time with respect to those treated with chemotherapy and/or RT, while cure rate trended to signification $(P=0.08)$. Interestingly, primary tumor staging was predictive for improved survival time but not for improved cure rate, while recurrent tumor staging was not predictive for any of them. In a general approach, among patients with recurrence, those who had primary tumors stage I-II, those having recurred after 6 months of initial treatment and those being amenable to surgical resection had better prognosis.

Kowalski et al., ${ }^{[4]}$ in a series of 513 patients with OSCC, observed an overall recurrence rate of $41.7 \%$, with $82(16 \%)$ patients showing a regional recurrence. Only 36 (44\%) patients were amenable to salvage surgery, with an overall survival after salvage surgery of $31 \%$ to $36 \%$, depending on the location of the recurrence in the ipsi- or contralateral neck. The authors found that patient's previously undergoing treatment of the neck experimented a poorer survival after recurrence than those not previously treated, and concluded that patients with neck recurrences have a poor prognosis despite salvage surgery.

In a series of 191 patients receiving curative intended surgery for SCC of the oral cavity, Lin et al. ${ }^{[1]}$ isolated 56 patients with recurrence, for whom salvage surgery was performed. By defining "early recurrence" as a localized tumor less than $4 \mathrm{~cm}$, without bone invasion in the computed tomography (CT)-scan, and "late recurrence" as a tumor larger than $4 \mathrm{~cm}$ with bone invasion that presented as a lymph neck node or a diffuse invasion in the CT scan, they found a 5-year disease-free survival rate of $24 \%$, with $32 \%$ of patients free of disease if an early recurrence was detected, in comparison to only $16 \%$ of patients free of disease if a late recurrence was treated. They also reported an acceptable overall 5-year survival rate of $60 \%$ for early recurrences, in contrast to $38 \%$ if recurrences were late.

Agra et al. ${ }^{[5]}$ in 2006, studied 246 patients with recurrent SCC of the oral cavity and oropharynx who underwent salvage surgery from a single institution. They found a statistical significant better 5-year overall survival in favor of: (1) early (I/II) (43.6\%) versus late recurrent clinical tumor, node, and metastasis stages (III/IV) (29.1\%), $P=0.027$; (2) disease free interval more than 1 year (42.1\%) versus less than one year $(26.7 \%), P=0.023$; and (3) previous treatment by surgery alone (39.3\%) versus surgery followed by RT $(26.1 \%)$ or RT alone $(25.3 \%), P=0.028$. There were no differences in relation to survival according to the period of admission, sex, age, type of recurrence, and status of surgical margins. Patients with recurrent cancer of the oral cavity showed a higher 5-year overall survival rate than patients with recurrent oropharyngeal cancer (33.6\% vs. 25.6\%), although this difference was not 
statistically significant $(P=0.226)$. Similarly, Koo et al. ${ }^{[6]}$ in a series about 127 patients with OSCC observed a $28 \%$ overall recurrence rate, with a $12 \%$ local recurrence, $13 \%$ regional recurrence, and $2 \%$ loco-regional. They reported a 5-year overall survival rate of $38 \%$ and the mean interval free of disease higher taller than in $18 \%$.

In a well-known study by Brown et al. ${ }^{[7]}$ about a series of 462 patients with OSCC treated by surgery followed or not by postoperative RT, they found an overall recurrence rate of $21 \%$, with a $10.4 \%, 7.35 \%$ and $3.46 \%$ of local, regional and loco-regional recurrence rates, respectively. They wanted to study the hypothetic benefit of postoperative RT in the group of patients at intermediate risk of recurrence, and observed that a significant higher proportion of patients undergoing adjuvant RT had locoregional recurrence (24\%) compared to those treated by surgery alone (15\%). They also found an improved salvage rate for recurrent disease in the surgery alone group (53\%) in comparison to the postoperative RT group (13\%).

Liao et al. ${ }^{[8]}$ in a series of 272 recurrent OSCC patients, found an overall recurrence rate of $28.5 \%$, with a local recurrence of $48.9 \%$ and a regional recurrence of $51.1 \%$. They observed that the cutoff point at 10 months from the initial treatment has the worst prognosis in terms of 5-year disease-specific survival (DSS) and overall survival (OS). They found that a late-relapse was associated with better survival than an early-relapse occurring within the first 10 months after primary treatment. Considering treatment in patients with early-relapsed OSCC, a significant benefit was demonstrated for salvage treatment (salvage surgery with or without RCT), in terms of both 5-year DSS and OS. Similarly, in patients with a late-recurrence OSCC, a significant improvement in both 5-year DSS and OS rates were observed for salvage therapy. It is interesting to note that salvage surgery was significantly better than salvage RCT for patients with late-relapsed OSSC but not for early-relapsed OSCC.

In 2008, Lim and $\mathrm{Choi}^{[9]}$ found recurrences of OSCC to appear in $21 \%$ of the patients with $\mathrm{T} 1$ and $\mathrm{T} 2$ tumors primarily treated with surgery alone, with $31 \%$ and $50 \%$ local and regional recurrences rates, respectively. They encountered a $36 \%$ OS rate for recurrent patients following salvage surgery. This recurrent rate is very similar to that reported by Brown et al. ${ }^{[7]}$ and also by Kernohan et al. ${ }^{[10]}$ with a $22 \%$ recurrence rate. These authors also reported a quite high OS of $50 \%$ for recurrent patients. Meanwhile, in a short series by Sklenicka et al.. ${ }^{[1] \mid}$ in 2010, they found a $15 \%$ recurrence rate, with $67 \%$ of recurrent patients undergoing further salvage surgery and an estimated 5-year disease-free survival of $48 \%$ for the whole series.

Kostrzewa et al., ${ }^{[12]}$ in a series of 72 recurrent OSCC patients that underwent salvage surgery, observed a $44 \%$ OS rate. These authors did not encounter a significant association between OS following salvage surgery and restaging after recurrence or margin status following surgical salvage. Conversely, they demonstrated a significant association between survival and time to recurrence, showing that recurrences within the first 6 months from the primary treatment had a worse prognosis.

According to Goto et al., ${ }^{[13]}$ in a series of 69 recurrent OSCC, the 5-year OS rate for those patients undergoing salvage surgery ranged from $86 \%$ in recurrent stage I or II to $48 \%$ in recurrent stage III or IV. Their multivariate analysis identified extra-capsular spread (ECS) as an independent prognostic factor for OS following salvage surgery, with patients presenting ECS at salvage surgery having a $37 \%$-year OS rate, in contrast to $78 \%$ for those do not presenting ECS.

\section{Overall results from the systematic review}

Several articles in the primary literature search evaluated recurrence and overall survival rates in relation to "head and neck cancer" or "squamous cell carcinoma" of the "upper aerodigestive tract", including oral cavity, oropharynx, hypopharynx and larynx subsites. As categorization was not always performed by the authors and results specifically dealing with OSCC were not either supported, these series were systematically excluded from our study. Thus, only those series specifically dealing with recurrence and overall survival at the oral cavity and/or oropharynx were selected and included for the analysis [Table 1].

From the finally analyzed 13 articles, a recalculation of the values within selected variables was performed. For their calculation, only those articles with available data in relation to a specific variable were selectively chosen. A total number of 1,692 patients with recurrent OSCC were included from the author's selection, ranging from 16 patients corresponding to the lower series to 434 patients from the largest one. The recalculated overall recurrence rate from the meta-analysis was $26 \%$ (range: $15-41.7 \%$ ), with a mean $47.3 \%, 35.1 \%$ and $10.9 \%$ of local, regional and loco-regional recurrences, respectively.

Except for a single paper, the 5-year OS rate was present in all selected papers. Regarding the survival expressed in terms of 5-year OS rate, a mean value of $40.2 \%$ (range: 37.5 $42.9 \%$ ) was obtained from the meta-analysis. Three particular series showed their results concerning survival in terms of categorization upon early stage (I/I) or advanced stage (III/ IV), and thus two values for the variable 5-year OS rate were provided. This could explain the observed range of survival rates between $37.5 \%$ and $42.9 \%$.

\section{DISCUSSION}

Survival of recurrent OSCC patients

Although some classical studies ${ }^{[14,15]}$ advocated for 
the use of only palliative treatment in recurrent oral cancer, mainly in advanced cancer previously treated with surgery and radiotherapy, actually a main role for curative treatment is advocated, basically by means of salvage surgery. In a meta-analysis by Goodwin et al., ${ }^{[16]}$ a 5 -year overall survival rate of $43 \%$ was reported for recurrent SCC of the oral cavity, while a $26 \%$ was referred for recurrent SCC of the oropharynx. In the study by Agra et al..$^{[5]}$ the overall 5-year survival rate was $32.3 \%$, with a $33.6 \%$ for the SCC of the oral cavity and a $25.6 \%$ for SCC of the oropharynx. Even, for advanced clinical stage recurrences (rCS III/IV), an overall 29.1\% 5-year overall survival was found. Zafereo et al. ${ }^{[17]}$ found a 3 -year and 5-year OS rates for salvage surgery of $42 \%$ and $28 \%$, respectively, being favorable patients for such a treatment: (1) youngers; (2) long disease-free interval after primary treatment; (3) small recurrent tumors for which is possible to obtain negative surgical margins; and (4) no recurrent neck disease. All these OS rates illustrate that, even for advance recurrent OSCC, salvage surgery is still an option with curative intention for many patients.

Prognostic factors for survival are not similarly considered in all the studies. While some authors ${ }^{[3]}$ did not find a relation between the clinical stage of the recurrence and survival, others have encountered a significant association among them. Agra et al. ${ }^{[5]}$ showed this association in the univariate as well as in the multivariate analysis. Goodwin et al. ${ }^{[16]}$ found that the clinical stage of the recurrent tumor was the most significant predictor of survival. Interestingly other authors ${ }^{[13]}$ have found the ECS as the most powerful prognostic factor for surveillance, even superior to the stage of the recurrence.

Also, recurrences before 1 year after primary treatment seem to have worse prognosis. This is somehow a common topic, as Liao et al., ${ }^{[8]}$ in their series of 272 recurrent OSCC, have also checked that early recurrences before 10 months after primary treatment had a significant worse prognosis in terms of 5-year DSS and OS than late-relapsed OSCC. Similarly, Zafereo et al., ${ }^{[17]}$ for recurrent SCC of the oropharynx, observed that a disease-free interval after primary treatment was a critical factor in predicting the success of salvage surgery. In concordance, Agra et al. ${ }^{[5]}$ showed that patients recurring less than a year before primary treatment had a significant worse prognosis.

While some authors ${ }^{[3,15]}$ have suggested that patients with advanced III/IV recurrent tumors that have received previous surgery followed by RT, based on poor prognosis, should only receive palliative treatment, this asseveration is no longer supported in the light of the results from the last decade. In fact, there are several studies $^{[5]}$ which indicate from multivariate analyses that previous treatment is not a predictor of survival anymore, and so advanced recurrent OSCC patients cannot be excluded from salvage surgery if general conditions and resectability criteria allows for it, as 5-year OS is higher than $30 \%$. In the light of the results from the present meta-analysis, with a mean 5 -year OS rate upper than $40 \%$, if candidates, salvage surgery is strongly recommended for treating recurrent OSCC patients.

In concordance with several other studies, Liao et al.$^{[8]}$ found that better prognosis was achieved for local recurrence in comparison to cervical recurrence. In concordance with it, Lim and Choi ${ }^{[9]}$ also observed that although good salvage was accomplished in local recurrences, a poor salvage rate was found in cases of cervical nodal recurrences, especially if being associated with a neck level IV recurrence. Goto et al. ${ }^{[13]}$ was coincident in asseverating that node involvement at levels IV or $\mathrm{V}$ was predictive for a decreased 5-year OS rate.

Besides several prognostic tumor-related factors, the influence of primary treatment modality in the outcome of recurrent OSCC patients has also been investigated. In relation to this, the study by Brown et al. ${ }^{[7]}$ demonstrated that the administration of postoperative RT negatively influenced prognosis and recurrence rate in patients at intermediate risk of recurrence that underwent further salvage surgery because of relapse. Goto et al. ${ }^{[13]}$ also demonstrated several prognostic factors being predictive for a worse 5-year OS after salvage surgery, such as advanced stages (III and IV) recurrent tumors, two or more positive cervical lymph nodes, positive cervical lymph nodes at levels IV or V, ECS, and diseasefree interval form primary treatment minor than a year.

\section{The role of salvage surgery}

Traditionally, in relation to resectability criteria, many concerns about the possibility for real resection of recurrent oral SCC and related reconstructive options made salvage surgery scarcely indicated. Nowadays, advances in surgical approaches, technology, microsurgical reconstruction, and also surgeons' training have extended indications for salvage surgery, even for patients with advanced recurrent clinical stages III/IV.

As pointed out by Liao et al. ${ }^{[8]}$ salvage therapy is of benefit for patients with early-relapsed OSCC in terms of both 5-year DSS and OS. Interestingly, these authors could not find a significant difference for salvage surgery compared with salvage RCT, regardless of whether patients have a local or a regional recurrence. In contrast, the benefit of salvage surgery over salvage RCT was clearly stated for patients with late-relapsed OSCC, in which recurrence appeared 10 months later than primary treatment. In relation to the benefit of salvage surgery, Zafereo et al. ${ }^{[17]}$ revealed that patients who underwent salvage surgery had a significantly higher 3-year OS rate than patients who underwent nonsurgical treatments (excluding patients who received supportive care), such as re-irradiation or brachytherapy or chemotherapy. 


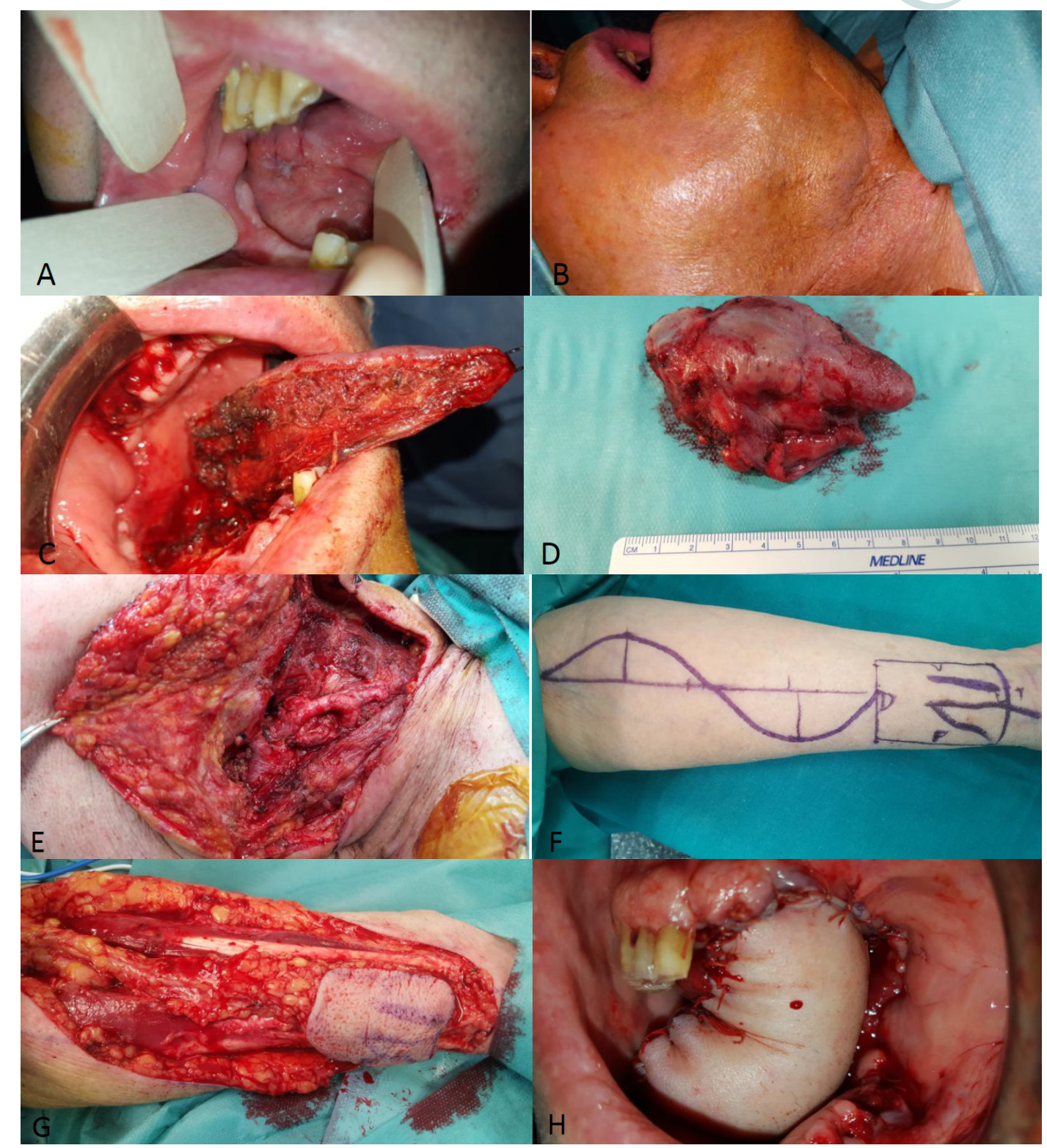

Figure 1: Recurrent oral squamous cell carcinoma (OSCC) patient 1. (A) Local recurrence of OSCC in the right side of the tongue; (B) regional recurrence in right cervical level II; (C) intraoperative hemi-glossectomy; (D) intraoperative view of the resected specimen; (E) classical radical neck dissection; (F) design of the "Iberic graft" technique for reconstruction of the defect with a radial forearm free flap (RFFF); (G) RFFF harvesting; (H) intraoral view of the reconstruction, with the RFFF showing arterial bleeding and viability

Actually, salvage surgery is being considered as the best treatment in recurrent OSCC, especially in those patients in which previous radiotherapy has been administered as part of the primary treatment, because of the toxicity of re-irradiation [Figure 1]. Re-irradiation in SCC of the aero-digestive tract has been reported to have an overall 5 -year survival rate up to $20 \%$, while chemotherapy is clearly discouraging. ${ }^{[5]}$ The real fact with many recurrent OSCC patients is that RT has already been administered as part of the primary treatment, in most of the cases as an adjuvant therapy to primary surgery, and re-irradiation with its inherent toxicity is not tolerated by the patient.

Besides, the main concern with respect to the use of RT is the increased morbidity, such as risk of osteo-radionecrosis and caries, worseness of speech and swallowing, and xerostomia, among others. Considering that some authors $^{[7]}$ have found a loco-regional recurrence rate of only $18 \%$ for patients at intermediate risk of recurrence [close surgical margins ( $>1 \mathrm{~mm}$ and $<5 \mathrm{~mm}$ ) and/or positive neck disease without ECS], and that $82 \%$ of them may not benefit from adjuvant RT, the clinician must reconsider the systematic administration of postoperative RT in those patients without cervical ECS or an involved margin ( $<1 \mathrm{~mm}$ from the margin).

In our experience from a single institution (University Hospital Infanta Cristina, Badajoz, Spain) treating SCC of the oral cavity and oropharynx by surgery in a population area of one million and five hundred thousand inhabitants (Extremadura, Spain), salvage surgery is the best option for recurrent patients, even in 


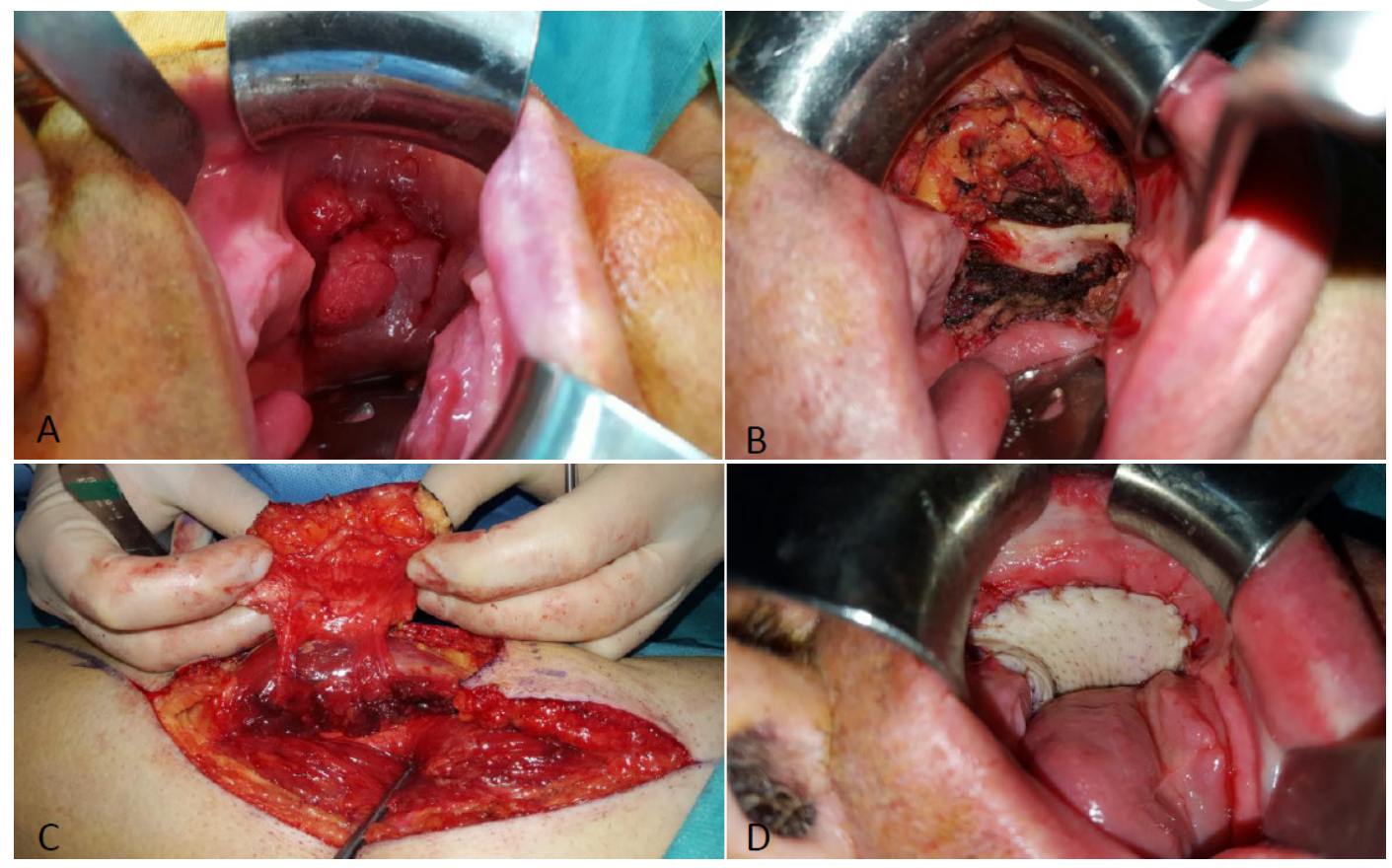

Figure 2: Recurrent oral squamous cell carcinoma (OSCC) patient 2. (A) Local recurrence of OSCC in the left buccal mucosa and retromolartrigone; (B) intraoral view of the defect after resection with margins; (C) harvesting of the anterolateral thigh flap; (D) intraoral view of the reconstruction with the anterolateral thigh flap covering defect in the buccal mucosa and retromolartrigone
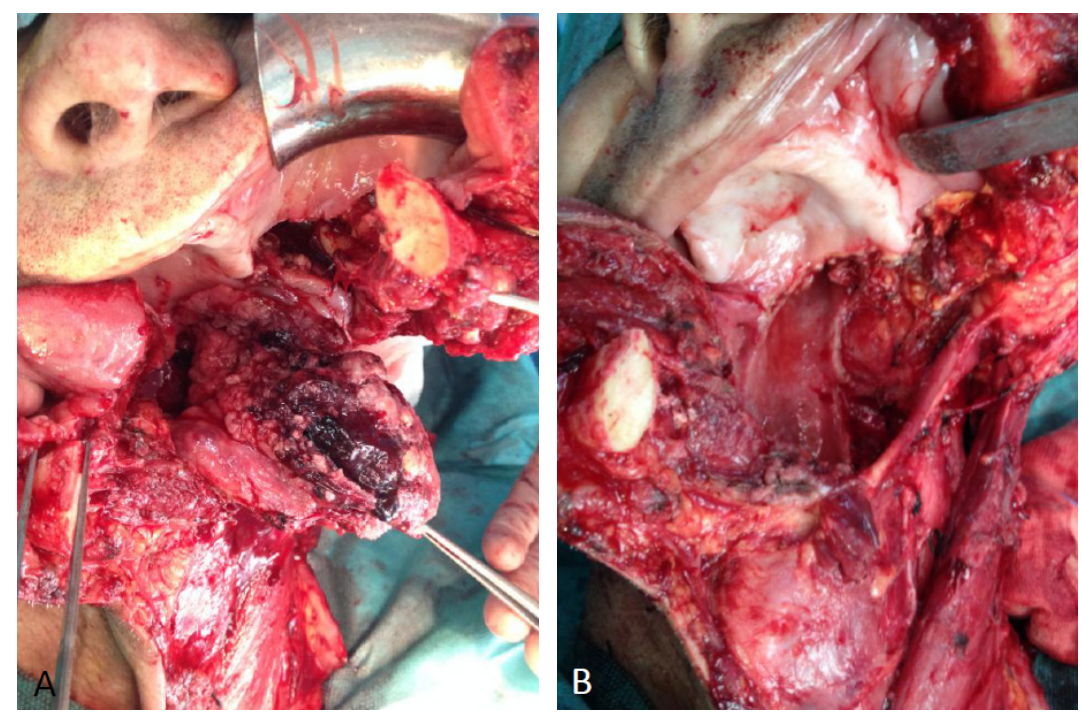

Figure 3: Recurrent oral squamous cell carcinoma (OSCC) patient 3. (A) Loco-regional recurrence of OSCC in the tongue. Intraoperative view of the resection and classical radical neck dissection; (B) intraoperative view following resection

advanced stages, if feasible [Figure 2]. We only reserve re-irradition (if toxicity tolerated), chemotherapy or supportive care for those patients with unresectable or inoperable recurrent tumors, in whom a radical surgical surgery is not warrantied and/or a general anesthetic procedure is an extremely risky intervention.

\section{Complications following salvage surgery}

One of the major concerns regarding performance of salvage surgery for the recurrent patients is the high rate of complications. Agra et al. ${ }^{[5]}$ reported a $37 \%$ overall complication rate and a mortality of $2 \%$ for salvage surgery, most of complications being wound infection, wound dehiscence and/or flap necrosis, and oro-cutaneous fistulas. A major complication such as the rupture of the carotid artery was only observed in $0.5 \%$ of the cases. Lin et al. ${ }^{[1]}$ reported an overall complication rate of the salvage group of $60.7 \%$, which was higher than that for patients primarily treated with surgery $30.4 \%$. However, no significant differences in terms of major complications were observed. This consideration is based on a myriad of recurrent tumors, with early and late stages mixed together.

Considering that for these authors, early recurrent tumors did not involve bone and/or neck, it is my believing, 
based on our own experience in treating advanced recurrent tumor with neck involvement, that the major complication rate may be significantly different when only a local recurrence is surgically treated or by contrast when a loco-regional or regional recurrence with neck involvement is managed [Figure 3]. Most of our recurrent patients have been previously treated by primary surgery with local excision and cervical neck dissection followed or not by loco-regional radiotherapy. The effect of prior operation and/or irradiation is determinant in the absence of anatomical layers or boundaries, which makes salvage surgery of the neck completely different from that performed in the primary treatment. Even, it is not uncommon among our recurrent patients to encounter advanced tumors in which diffuse invasion is present, with invasion of major vascular structures such as the internal jugular vein and surrounding musculature, which subsequently makes surgery much more difficult and hazardous, with potential severe complications such as massive bleeding from big cervical vessels.

Thus, based on previous series and on our own experience, we believe that: (1) overall complication rate for salvage surgery is higher than that for primary surgery; (2) major complications rate does not seem to be statistically different for salvage surgery; and (3) major complication rate and postoperative mortality from salvage surgery does not seem to differ from that observed for advanced stages III/IV primary OSCC, but this should not be extensible to early stage tumors (I/II) treated primarily by surgery, in which major complications are being expected in a lower rate.

Also, the influence of long-term radiation toxicity derived from its postoperative administration in the primary treatment and/or following salvage surgery may determine the appearance of xerostomia, osteo-radionecrosis of the jaws or restricted mouth opening and lingual movements, which may add its deleterious effects to those derived from surgery. Even, despite the performance of free flap reconstruction, up to $25-50 \%^{[16]}$ of the patients may require a permanent gastrotomy because of feeding impairment, although these considerations concerning sequelae are beyond the scope of the present paper.

In summary, with an overall $26 \%$ recurrence rate for OSCC despite primary treatment with surgery followed by postoperative radiotherapy, the clinician must be firmly aware of recurrence, which is mainly a local relapse in more than $47 \%$ of the cases, followed by a regional recurrence in $35 \%$ and a combined loco-regional relapse in almost $11 \%$ of the cases. While chemotherapy is clearly discouraging and radiotherapy has only been reported to be curative in no more than a $20 \%$, salvage surgery is the best option for the treatment of recurrent OSCC patients, also for those with advanced disease, with a mean 5-year overall-survival rate of more than $40 \%$ of the cases, showing acceptable morbidity and good functional results if reconstructive microsurgical techniques are used.

\section{Financial support and sponsorship}

Nil.

\section{Conflicts of interest}

There are no conflicts of interest.

\section{REFERENCES}

I. Lin YC, Hsiao JR, Tsai ST. Salvage surgery as the primary treatment for recurrent oral squamous cell carcinoma. Oral Oncol 2004;40:183-9.

2. Gleich LL, Ryzenman J, Gluckman JL, Wilson KM, Barrett WL, Redmond KP. Recurrent advanced (T3 or T4) head and neck squamous cell carcinoma: is salvage possible? Arch Otolaryngol Head Neck Surg 2004; I30:35-8.

3. Schwartz GJ, Mehta RH, Wenig BL, Shaligram C, Portugal LG. Salvage treatment for recurrent squamous cell carcinoma of the oral cavity. Head Neck 2000;22:34-4I.

4. Kowalski LP. Results of salvage treatment of the neck in patients with oral cancer. Arch Otolaryngol Head Neck Surg 2002; 1 28:58-62.

5. Agra IM, Carvalho AL, Ulbrich FS, de Campos OD, Martins EP, Magrin J, Kowalski LP. Prognostic factors in salvage surgery for recurrent oral and oropharyngeal cancer. Head Neck 2006;28: I07-I3.

6. Koo BS, Lim YC, Lee JS, Choi EC. Recurrence and salvage treatment of squamous cell carcinoma of the oral cavity. Oral Oncol 2006;42:789-94.

7. Brown JS, Blackburn TK, Woolgar JA, Lowe D, Errington RD, Vaughan ED, Rogers SN. A comparison of outcomes for patients with oral squamous cell carcinoma at intermediate risk of recurrence treated by surgery alone or with post-operative radiotherapy. Oral Oncol 2007;43:764-73.

8. Liao CT, Chang JT, Wang HM, Ng SH, Hsueh C, Lee LY, Lin CH, Chen $\mathrm{IH}$, Huang SF, Cheng AJ, Yen TC. Salvage therapy in relapsed squamous cell carcinoma of the oral cavity: how and when? Cancer 2008; I I2:94103.

9. Lim YC, Choi EC. Surgery alone for squamous cell carcinoma of the oral cavity: survival rates, recurrence patterns, and salvage treatment. Acta Otolaryngol 2008; I28: I |32-7.

10. Kernohan MD, Clark JR, Gao K, Ebrahimi A, Milross CG. Predicting the prognosis of oral squamous cell carcinoma after first recurrence. Arch Otolaryngol Head Neck Surg 2010;136:1235-9.

II. Sklenicka S, Gardiner S, Dierks EJ, Potter BE, Bell RB. Survival analysis and risk factors for recurrence in oral squamous cell carcinoma: does surgical salvage affect outcome? J Oral Maxillofac Surg 2010;68:1270-5.

12. Kostrzewa JP, Lancaster WP, Iseli TA, Desmond RA, Carroll WR, Rosenthal EL. Outcomes of salvage surgery with free flap reconstruction for recurrent oral and oropharyngeal cancer. Laryngoscope 2010;120:267-72.

13. Goto M, Hanai N, Ozawa T, Hirakawa H, Suzuki H, Hyodo I, Kodaira T, Ogawa T, Fujimoto Y, Terada A, Kato H, Hasegawa Y. Prognostic factors and outcomes for salvage surgery in patients with recurrent squamous cell carcinoma of the tongue. Asia Pac J Clin Oncol 20|6;| $2:|4|-8$.

14. Cooney TR, Poulsen MG. Is routine follow-up useful after combinedmodality therapy for advanced head and neck cancer? Arch Otolaryngol Head Neck Surg 1999; I25:379-82.

15. Goodwin WJ Jr. Salvage surgery for patients with recurrent squamous cell carcinoma of the upper aerodigestive tract: when do the ends justify the means. Laryngoscope 2000; I 10:1-18.

16. Dziegielewski PT, Ho ML, Rieger J, Singh P, Langille M, Harris JR, Seikaly $H$. Total glossectomy with laryngeal preservation and free flap reconstruction: objective functional outcomes and systematic review of the literature. Laryngoscope 2013; $23: \mid 40-5$.

17. Zafereo ME, Hanasono MM, Rosenthal DI, Sturgis EM, Lewin JS, Roberts DB, Weber RS. The role of salvage surgery in patients with recurrent squamous cell carcinoma of the oropharynx. Cancer 2009; | 15:5723-33. 